\title{
THE MAGNETIC FIELD IN THE PERSEUS MOLECULAR CLOUD COMPLEX
}

\author{
A.A. GOODMAN and P.C. MYERS \\ Harvard-Smithsonian Center for Astrophysics, Cambridge, MA 02198, USA \\ P. BASTIEN Université de Montréal, Canada \\ R.M. CRUTCHER University of Illinois, USA \\ C. HEILES University of California, Berkeley, USA \\ I. KAZÈS Observatoire de Paris-Meudon, France \\ T.H. TROLAND University of Kentucky, USA
}

\section{Field Structure $\left(B_{\perp}\right)$}

In Figure 1, we present a map of the polarization of background starlight in the Perseus region (Goodman, Bastien, Myers, and Ménard 1989) superposed on contours of integrated ${ }^{13} \mathrm{CO}$ emission (Bachiller and Cernicharo 1986). The polarization vectors map the plane-of-the-sky field $\left(B_{\perp}\right)$, assuming as usual that the observed polarization is the result of selective extinction by magnetically aligned dust grains associated with the molecular clouds between the observer and background stars (e.g. Dolginov 1989).

The distribution of polarizations observed is bimodal. The first peak, centered at $71^{\circ} \mathrm{E}$ of $\mathrm{N}$ corresponds to a pattern of small $(\sim 1 \%)$ vectors observed to be almost aligned with the overall projected cloud axis, at $\sim 65^{\circ}$. The second peak, at $\sim 145^{\circ}$, is comprised of larger polarizations oriented almost perpendicular to the cloud axis. Thus there may be two distinct clouds, with different fields, along the line of sight to Perseus. Molecular line maps of $\mathrm{OH}$ and ${ }^{13} \mathrm{CO}$ emission (Bally, private communication) show significant velocity discontinuities between neighboring clouds, which also suggests multiple clouds.

\section{Field Strength Measurements $\left(B_{\|}\right)$}

We have carried out a series of $\mathrm{OH}$ and $\mathrm{HI}$ Zeeman observations. The results obtained in the region near the dark cloud Barnard 1 (B1) are summarized in Figure 2. The line-of-sight field strength $\left(B_{\|}\right)$as measured by $\mathrm{OH}$ Zeeman observations is strongest at the $(0,0)$ position of the map, which is also associated with the peak of an $\mathrm{NH}_{3}$ core (Bachiller et al. 1989), and with an IRAS source whose spectrum is typical of an embedded protostar (Beichman et al. 1986).

The measurements illustrated in Figure 2 are consistent with models of equipartion among magnetic, kinetic, and gravitational energy in self-gravitating molecular clouds (see Goodman et al. 1989 ; Myers and Goodman 1989; and references therein).

\section{References}

Bachiller, R. and Cernicharo, J. 1986, Astron. and Astrophys., 166, 283.

Bachiller, R., Martin-Pintado, J., Cernicharo, J., and Menten, K. 1989, in prep.

Beichman, C.A., Myers, P.C., Emerson, J.P., Harris, S., Mathieu, R.D., Benson, P.J., and Jennings, R.E. 1986, Ap. J., 307, 337.

Dolginov, A.Z., this volume.

Goodman, A.A., Bastien, P., Myers, P.C., and Ménard, F. 1989, in prep.

Goodman, A.A., Crutcher, R.M., Heiles, C., Myers, P.C., and Troland, T.H. 1989, Ap. J. (Letters), 338, L61.

Myers, P.C. and Goodman, A.A. 1989, this volume. 
Figure 1: An optical polarization map of the Perseus dark cloud complex superposed on ${ }^{13} \mathrm{CO}$ $(J=1-0)$ integrated intensity contours of 2,6 , and $10 \mathrm{~K} \mathrm{~km} \mathrm{~s}^{-1}$ from Bachiller and Cernicharo 1986. Note: The length scale shown assumes a cloud distance of $350 \mathrm{pc}$.

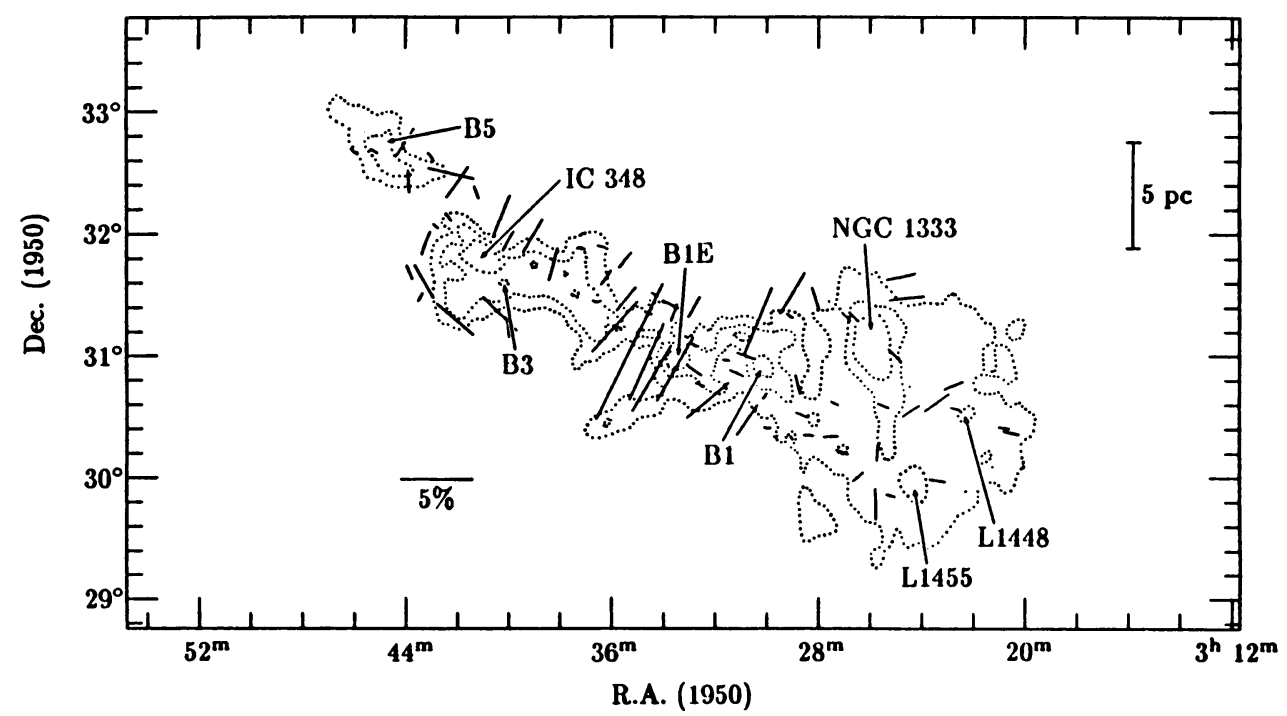

Figure 2: Contour Map of $\mathrm{OH} 1667 \mathrm{MHz}$ Emission in the region near Barnard 1 (B1). (Crosses indicate postions observed; FWHM beamsize for the mapping observations is $3^{\prime}$ (Arecibo $305-\mathrm{m}$ telescope).) The results of five separate long-integration Zeeman observations are indicated; circles indicate the FWHM beam for each observation. Stars indicate IRAS sources present in the region whose spectra are typical of embedded protostars. The coordinates of the observed positions are as follows- B1: $\alpha_{1950}=03^{h} 30^{m} 12.0^{\circ}, \delta_{1950}=30^{\circ} 57^{\prime} .6^{\prime \prime}(=(0,0)) ;$ P9: $\alpha_{1950}=03^{h} 30^{m} 12.0^{\circ}$, $\delta_{1950}=31^{\circ} 08^{\prime} 57^{\prime \prime}$; B1SW: $\alpha_{1950}=03^{h} 29^{m} 58.0^{\circ}, \delta_{1950}=30^{\circ} 54^{\prime} 26^{\prime \prime}$.

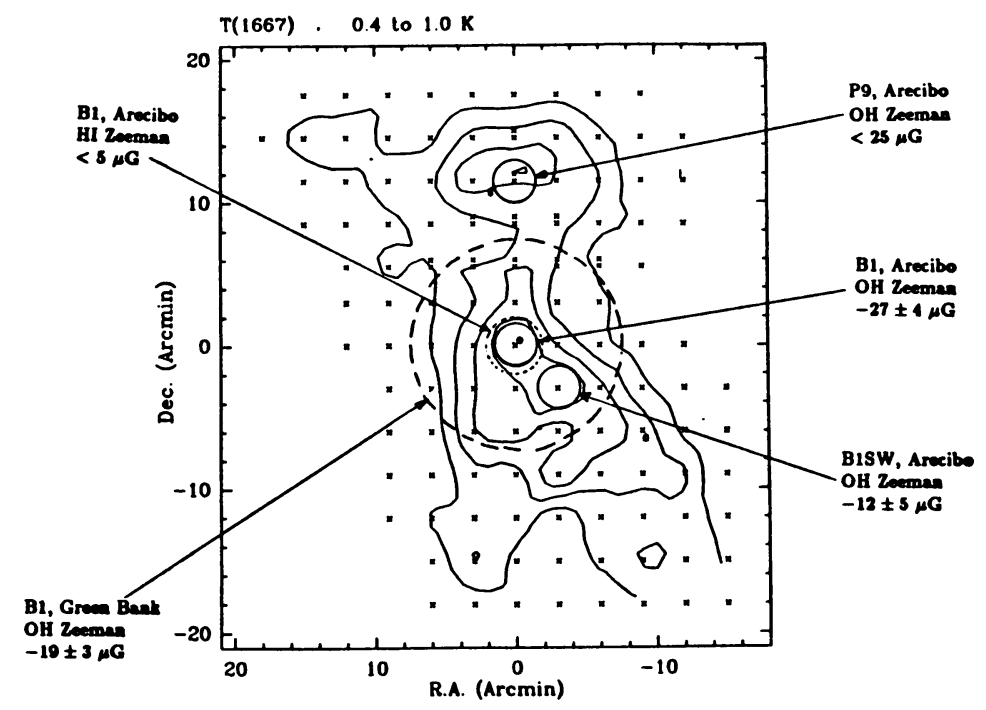

